\title{
Shaping Air Negative Ion Life Circles in the Coastal Space for Healthy Cities
}

\author{
Haotian XU, School of Architecture and Fine Art, Dalian University of Technology, China \\ Chenling WU, School of Architecture, Tianjin University, China
}

\begin{abstract}
Looking back on the mutual development of urban planning and public health, health has always been a topic that people cannot avoid. Nowadays, people pay more attention to health, and their demands for high-quality air in cities are becoming stronger. Air quality is an important factor affecting human health. Therefore, it is proposed to meet public health demands through planning interventions from the perspective of negative air ions. Air negative ions are a part of the air component and have functions such as medical care and air purification. As an important indicator to evaluate whether the urban air is good, it has a positive effect on public health. The coastal space has abundant health resources and contains a high concentration of negative air ions, which is an important space carrier to promote public health. Combining with the health effects of negative air ions, taking the influence of urban space layout on the concentration of negative air ions as the starting point, analyze the relationship between coastal space reserves and negative ion generation, urban spatial spread, and changes in negative ion concentration, spatial configuration and the acquisition of negative oxygen ions. On this basis, combined with public health needs, it is concluded that blue and green are used as the core organization function to build a high-negative ion living unit, and combined with the prevailing wind direction, the "wind-negative ion" channel is opened, the architectural space layout is optimized, and a variety of negative ion breathing areas are created. And other coastal area space optimization strategies, to achieve the optimization of the coastal space with negative air ions as the medium, and then achieve the effect of health promotion.
\end{abstract}

\section{Keywords}

Negative air ions, Public Health, Coastal Space, Urban Microclimate, Optimization Strategy

\section{Introduction}

\subsection{Urban planning and the development of public health}

Public health is closely related to urban planning. In the early 19th century, the outbreak of cholera in the UK caused a series of public health concerns. Public health problems "attributed to environmental problems rather than medical problems" and proposed the formulation of the "Public Health Act" (Jia, Liu, $\mathrm{Wu}, 2020)$. From the middle of the 19th century to the middle of the 20th century, public health began to use the "bacteria theory" theoretical system to explore the root causes of diseases and their control measures; at the same time, environmental determinism such as the garden city theory and the garden city movement tried to intervene in the material space Environment to deal with public health issues such as population gathering, housing renovation, functional zoning, and traffic congestion. Since the 1980s, with the development of the Healthy City Movement, urban planning and public health have been 
organically integrated, and the two have conducted in-depth discussions and conclusions on the concept, methods, and evaluation criteria of healthy cities. After entering the 21st century, the impact of the healthy city movement continues to expand. More scholars on public health issues have conducted more in-depth cross-over studies based on combining theories and methods of urban and rural planning and public health. With the global outbreak of new crown pneumonia in 2019, human settlements, public health, and public space planning have become the focus of continuous attention, especially the air quality in people's living environments.

\subsection{The public's demands on air quality}

With the rapid development of urbanization, the problem of environmental pollution has become increasingly prominent. Among them, air pollution has become the focus of public attention. Among the top ten factors affecting global health announced by the World Health Organization in 2019, the biggest environmental threat is air pollution. The inhalation of pollutants in the air by the human body can cause damage to the heart, lungs, and brain. Cancer, stroke, and heart and brain diseases caused by air pollution causes 7 million deaths each year. Air pollution is more serious in some developing countries, and $90 \%$ of deaths caused by air pollution occur in these countries (WHO Media centre, 2011).

Relevant studies in recent years have shown that negative air ions can purify the air to a certain extent and have the function of health care. The content of negative ions in the air is an important reference index for judging fresh air. At present, most of the research on negative ions is concentrated in the fields of ecological environment, forestry plants, etc., such as comparative analysis of the difference of air negative ions in different regions, the correlation between the changes of air negative ions and the hierarchical configuration of plants such as trees, shrubs, and grass. In the use of parks and scenic spots, etc., there are relatively few studies on shaping the coastal space of healthy cities from the perspective of negative air ions. At the same time, air quality is also one of the important factors affecting public health. Therefore, the concept of health promotion should be adopted from multiple angles and directions. In the construction of healthy cities, the use of urban spatial planning to intervene in the urban local microclimate, energy structure, oxygen source green space, human activities, and other factors (Qian, Pan, $\mathrm{Xi}, 2016)$, to increase the concentration of negative ions to promote the effect of health care, to improve the level of public health.

\subsection{Urban planning and the development of public health}

Human health is based on the dynamic balance between man and the natural environment. The coastal water area is the area where man and nature are most closely connected, and it has a direct impact on human health. Since the Industrial Revolution, the waterfront area has gradually become the most vibrant area in the city. Since the 1950s, the global adjustment of industrial structure has led to the decline of water transport ports, followed by large-scale development and construction of the waterfront. In the post-industrial era, the value of "people-oriented" has returned, and the public's demand for recreation and social communication in the open waterfront space has increased, which has once again aroused global attention to waterfront construction (Chu, 2010). In addition, studies have shown that due to the free phenomenon of water movement, the concentration of negative oxygen ions and negative water ions in the air in the coastal water area is higher than that in other areas. It can be seen that the waterfront district has unique natural scenery and health resources, and is one of the best areas to promote public health activities. However, the current rough development of the urban waterfront in many areas has caused various unhealthy phenomena in the construction of the waterfront, such as environmental pollution, poor air quality, messy shapes, and insufficient vitality, resulting in the urban public not being able to enjoy the waterfront in a normal and healthy manner. Water public space has seriously affected the physical and mental health of the urban public. Therefore, optimizing the urban 
coastal space environment to meet the public's demand for clean air and healthy living space has become an urgent problem to be solved today.

\section{Overview of air negative ions and public health in coastal space}

Air negative ions are free electrons separated by air molecules under the action of cosmic rays, sun rays, electromagnetic waves, ocean waves, waterfalls, and various meteorological activities. These free electrons combine with neutral molecules in the air to form negatively charged ions (Lin, Song, Zhao, 2006). The coastal area has the innate advantage of a high concentration of negative ions. With the fluctuation of seawater, the water droplets peel off through the interaction of forces during the impact and fall process, forming a fine water mist. When the fine water mist leaves the surface of the water droplet, it carries a negative charge, thus forming negative ions. As a result, the continuous splitting of water molecules produces a large number of negative ions. At the same time, plants around coastal areas can accelerate the positive and negative ionization of air molecules through the "photoelectric effect" and "tip discharge", release volatile substances such as "Phytoncide", which can continuously stimulate negative air ions.

The concentration of negative ions in the air in the coastal area is relatively high, which has purifying and health-care effects on the regional atmospheric environment and human functions respectively, thereby promoting human health. First, increasing the concentration of negative air ions can reduce the hazards of air pollution. Air pollution caused by the discharge of inhalable particles, sulfur dioxide, and other pollutants can affect health (WHO Media centre, 2011). The negatively charged nature of air negative ions enables it to combine with positively charged particles such as bacteria, dust, smoke, etc. in the air to gather precipitation and purify the air. According to observations, when the concentration of negative ions in the air reaches $20,000 \mathrm{~N} / \mathrm{cm} 3$, the amount of floating dust in the air can be reduced by more than 98\% (Lin, Song, Zhao, 2006); Second, the environment with a high concentration of negative oxygen ions has the effect of body health care. Oxygen is an indispensable element of life movement. Negative oxygen ions are one of the active species of oxygen, and their concentration is also closely related to human health. Studies have shown that when the concentration of negative oxygen ions is lower than 20 $\mathrm{N} / \mathrm{cm} 3$, people will feel tired and dizzy; when the concentration is between $1000-10000 \mathrm{~N} / \mathrm{cm} 3$, they feel calm and stable; when the concentration of negative oxygen ions is above 100,000 , Negative oxygen ions can also play a role in preventing and curing diseases such as eliminating fatigue, regulating nerves, etc. (Lin, Song, Zhao, 2006), therefore, negative air ions are also known as "air vitamins."

\section{The actual impact of urban construction on the distribution of air anion concentration}

Air negative ions can not only purify the air but also promote human health. The development of negative air ion resources is a scientific way to build a healthy city. The average concentration of air anions on land is lower than the average concentration of air anions in ocean areas, but the distribution is uneven. There are differences in the level of negative air ions between cities and suburbs and different functional areas. The reasons for the above phenomena are not only related to regional altitude, seasonal changes, and other natural factors, but also the erosion of oxygen source green space during urban construction, changes in urban energy structure, and the influence of people's life behaviors also change the distribution of negative ions.

\subsection{Blue-green spaces and negative ion generation}

Vegetation and water system are the main sources of negative air ions. The concentration of negative air ions is positively correlated with biodiversity, green coverage, water area and flow velocity (Wang, Yu, 
Zhen, 2012). How to protect and optimize the urban green space system structure is the prerequisite for creating an urban environment with high negative ions. However, the encroachment and destruction of green land and rivers in the rapid urban development often make the scale of these negative ions generation sources less and less. Studies have shown that when the forest coverage rate reaches $35 \%$ $60 \%$, the air anion concentration is the highest, but when the forest coverage rate is less than $7 \%$, the negative oxygen ion concentration is only $40 \%-50 \%$ of the above situation (Editor-in-Chief of Beijing Forestry College, 1999). Moreover, the laying of a large amount of cement and asphalt pavement blocks the ionization source from the soil, thereby reducing the content of negative air ions (Meng, Zhang, 2004). In addition, the reduction of water area, lower flow rate, and habitat destruction caused by waterfront development such as land reclamation, river reconstruction, and docking will also affect the level of negative air ions to varying degrees.

\subsection{Urban spatial layout and changes in negative ion concentration}

The coastal area is an area where the interaction between land, ocean, and atmosphere is relatively active. The abundant air anions in the coastal area can "charge" public health, but there are many factors that affect the distribution of air anions in the coastal area. The coastal space environment is closely related to the local microclimate, such as the regional temperature, humidity, wind speed, and other microclimates. At the same time, the coastal area's spatial layout, rapid expansion and construction, changes in energy structure, and human flow activities will also affect the distribution of air negative ion concentration has an impact.

On the one hand, because the concentration of negative air ions is also related to the local microclimate, including temperature, wind, relative humidity, etc., the formation of the atmospheric boundary layer, regional climate, heat island effect, and other phenomena are closely related to the diffusion and circulation of air, It also has a certain impact on the distribution of negative air ions (Yin, 2018).

On the other hand, the urban center is densely populated. Excessive waste gas, dust, aerosols composed of various particles and other pollutants released by industry and automobile exhaust emissions, urban construction, etc. will combine with air negative ions and settle to make small ion concentration. Decrease rapidly, thereby reducing the concentration of negative air ions. The detection of air anion levels in different functional areas has shown that the air anion content in industrial areas and busy commercial traffic areas are significantly lower than that in urban coastal and green areas where traffic flow is relatively stable (Qian, Pan, Xi, 2016; Ling, Jayaratne, Morawska,2010).

\subsection{Community space configuration and negative oxygen ion acquisition}

The air anion concentration has a law of attenuation with the space environment, and the farther away from the anion source, the less anion (Zhang, 1994). From the perspective of health promotion, the evaluation of the concentration of negative air ions not only depends on the number of negative ions per unit area but also relates to how easy it is for citizens to obtain negative air ions.

The community environment is the environmental factor most closely related to the health of citizens. The relationship between its green water system and residents' residences, medical and healthcare resources, and the relationship between building layout and dominant wind direction will all have an impact on the spread of negative air ions in the community. On the one hand, the green water system in and around the community is not only an outdoor activity place for residents but also a major air negative ion breathing area. The convenient connection with residences and medical care resources is an important way for residents to obtain negative oxygen ions; On the one hand, atmospheric movement will drive the flow of air and promote the diffusion of negative air ions. Community planning, site selection, and building space layout will change the local wind environment and affect the concentration of negative air ions in the community. For example, the air anion concentration in the downwind 
community is generally higher (Li, Lu, 2004), and the air anion concentration in the high-density residential area at the bottom is higher than that in the high-rise residential area (Wang, Yu, Ji, 2013).

\section{Construction strategy of negative ion life circle in urban coastal space}

The negative ion life circle refers to the urban living unit with a high concentration of negative ions in the city constructed with air negative ion sources such as urban parks, blue-green corridors, forest tourist areas, waterfalls, and waterfront scenic spots as the core. Through the adjustment of the functional structure, the optimization of the blue-green system, and the construction of the space network, the concentration of the air negative ion generation area is increased, and the spatial connection with the surrounding areas and civil activities is strengthened. The main way of healthy city design under the relationship.

\subsection{The blue-green space is the function of nuclear organization, building a living unit}

\section{with a high negative ion concentration}

The green water system is the main source of negative air ions. By adjusting the spatial structure, optimizing the coastal water, landscaped green space, and other ecological systems, and providing the public with convenient and accessible, high-quality negative ion living units are important foundations for improving and optimizing the spatial environment.

\subsubsection{Optimizing the layout of waterfront space}

Urban waterfront space is an outdoor activity space centered on people, water, and cities. It needs to meet people's needs in social communication, mental health, physical health, and so on. At the same time, it is necessary to strictly control the development of waterfront space. Waterfront space resources are very limited. It is necessary to strictly delimit the urban blue line boundary, protect the healthy resources of waterfront space, clarify the land ownership of waterfront space, and prohibit other land ownership from breaking through the blue line. As for the boundary of the line, a certain buffer space should be reserved for the waterfront space.

The spatial arrangement of regional water bodies is generally divided into two types, namely, the water body space of Huachi and the water body space of lakes. It is generally recommended to be arranged in areas where the public gathers, such as square parks, public open spaces in residential areas, etc., where the waterscape of the flower pond should be fluid, such as a fountain. According to research, it is found that in a waterfall environment, water molecules will be affected by the impact when the water drops. It is separated into atoms and combined with oxygen ions formed by ionization in the air to form a very high concentration of negative oxygen ions. The fountain is similar to a waterfall, and the concentration of negative oxygen ions can suddenly increase to a higher concentration when the fountain is turned on. Therefore, as many fountains as possible can be arranged in public gathering spaces, which can not only greatly increase the concentration of negative oxygen ions in the air and improve air quality, but also can beautify the environment and adjust the surrounding air quality. The opening time of the fountain is related to the moment of gathering and dispersing of people in the area. It can be designed by the time law of air anions in the Dalian coastal area. The fountain can be opened at noon when the air anion concentration is the lowest in the day to increase the anion concentration in space.

For the installation of static lakes and other water bodies, the riverside resources should be fully utilized, and hydrophilic platforms should be added at the important nodes of the riverside, preferably at the windward, so that the movement of the airflow caused by the physical changes of the water body can produce more The negative air ions, and help to improve the microclimate of the region. 


\subsubsection{Optimizing the layout of green space}

Green space plays an important role in improving and enhancing the comfort of the urban climate and the quality of the space environment. For example, green space can increase air humidity, improve air freshness, and reduce regional temperature.

In the layout of landscape greening, full consideration should be given to the selection and combination of vegetation types. On the one hand, the use of vegetation can achieve dust reduction and reduce the concentration of fine particles in the area. On the other hand, the tip discharge of plants can also produce a certain concentration of negative air ions.

In the layout of green space in the coastal area, the layout of green space to prevent fragmentation can be arranged with squares and open spaces as the center, connecting other green spaces or water bodies in the area, forming a systematic green space system ( $\mathrm{Chu}, \mathrm{Li}, \mathrm{Li}, 2012$ ). In addition, for the layout of the vegetation green space structure of the park square as much as possible, changing the layout of a single grassland and a single tree will not only increase the distribution of different types of vegetation and green space in the horizontal direction but also in the vertical direction. The grass is mixed and arranged to form a three-dimensional green space. This multi-level configuration helps to increase the concentration of negative ions in the area.

\subsection{Combining the prevailing wind direction to open up the "wind-negative ion" channel}

Taking the improvement of the coastal area's space environment as the starting point, creating a "windnegative ion" channel as a means to improve air quality and promote public health as the goal.

Atmospheric circulation promotes the distribution and diffusion of gas and also facilitates the migration of negative air ions. The "wind-negative ion" channel is a transfer medium that connects the highconcentration negative ion compensation space and the area where the wind resistance is small and needs to be compensated for negative ions. Urban parks, blue and green corridors, forest sightseeing areas, waterfalls, waterfront areas, etc. are all air negative ions. Compensation space.

When designing and arranging the "wind-negative ion" channel area, in the macro-scale of the city, it is necessary to protect the ocean shoreline, river system, dredge the urban greenway, suburban park, and ocean forest, create the urban area ventilation corridor, and make the ocean forest natural Highconcentration negative ions in ecological areas and suburban parks are transported to the interior of the city using wind as the medium.

At the macro-block spatial scale, the prevailing wind direction on the ground should be considered, and the downwind direction of construction areas with polluted air or planned construction areas should be avoided as much as possible, and the underlying surface of the "wind-negative ion" channel area should be controlled. The layout direction of roads and linear green spaces, the volume of buildings and other elements to prevent them from blocking the incoming airflow, to ensure the smooth flow and diffusion of airflow in the "wind-negative ion" channel, and then achieve the effect of dispersing air pollutants and purifying regional air.

\subsection{Optimizing the spatial layout of the building and create a variety of negative ion}

\section{breathing areas}

Taking the concentration of negative air ions as one of the reference indicators for constructing and evaluating healthy cities, after specific evaluations of healthy cities, the districts are optimized and managed according to the evaluation results. Then take blue and green as the core to optimize the space layout of the building within the scope of the land. For different building groups, the layout form and 
orientation will cause different ventilation phenomena. Therefore, controlling the opening method and spacing scale of the building is an effective way to promote the flow of negative ions in the air.

\subsubsection{Optimizing the layout of the building}

In terms of the architectural layout of the plots, the wind environment conditions of the rows and columns are better than those of the regular layout, and the ventilation environment of the enclosed layout and the mixed layout is relatively general.

For the layout of the building, when the windward side of the building is perpendicular to the incoming dominant wind, the distance between the front and rear of the building needs to be long-distance, so that the rear exhaust environment of the building group will not be affected by the front row of buildings. The outdoor ventilation effect of the row building can be increased by changing the opening method of the boundary building. Generally, there are two ways to increase the wind rate. One is to increase the size of openings between buildings, and the other is to increase the number of openings between buildings, that is, an opening is set between the continuous building groups in the air outlet area, or open space is set in the air outlet area, which is more conducive to the ventilation of the building group and ensures the smooth flow and diffusion of negative air ions (Liu, 2011), as shown in Figures 1 and Figures 2.

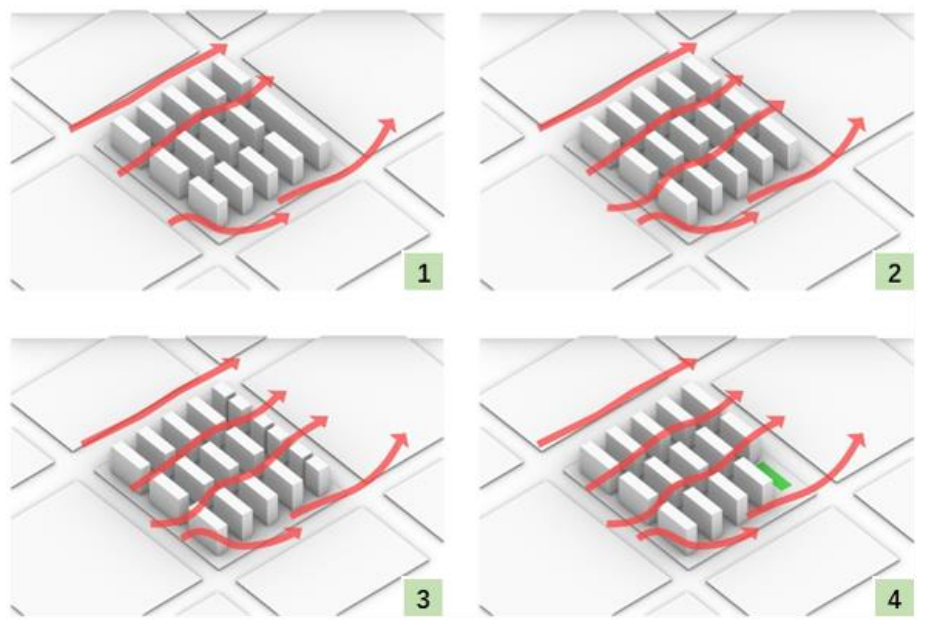

Figure 1. Analysis of space optimization process under the determinant layout. Source: Authors.
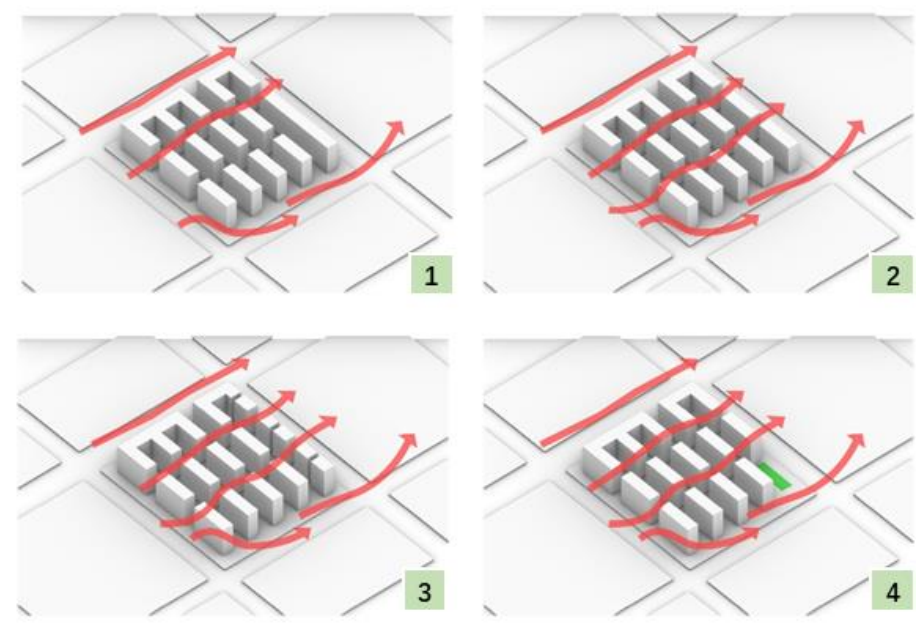

Figure 2. Analysis of space optimization process under the enclosed layout. Source: Authors. 


\subsubsection{Optimizing the architectural orientation design}

When building space layout, the orientation design of the building group should generally be considered. The angle of the building can be set reasonably in accordance with the prevailing wind direction so that the outdoor wind environment of the building group is smoother, and the area of the local quiet wind area is reduced, thereby improving the wind environment. The flow method of the outside wind has a great relationship with the layout angle of the building. When the airflow encounters the "obstacle" on the windward side of the building, it will generate a vortex (that is, the wind shadow area). When it reaches the rock, a vortex will flow down the rock. The size of the vortex is not only related to the length of the projection surface perpendicular to the wind direction on the windward side of the building but also related to the smoothness of the building's façade and the projection angle of the wind direction (Liu, 2011).

Architectural orientation mainly refers to the orientation design of the windward side of the building. The windward side is the interface where the building first affects the incoming wind. Therefore, the design of the windward side of the building is very important. The wind environment in the plot has a direct impact.

When the projection angle of the wind direction is $0^{\circ}$, the vertical design of the building group and the incoming wind direction will make the wind shadow area generated by the incoming wind on the leeward side of the building when it bypasses the windward side larger, making the rear building in the front row of buildings In the wind shadow area, it is not conducive to the air guidance of the building group; and when the wind direction is projected at a certain oblique angle, the wind shadow area generated by the leeward side of the building group is less, which is beneficial to the air guidance inside the residential area, as shown in Figure 3.

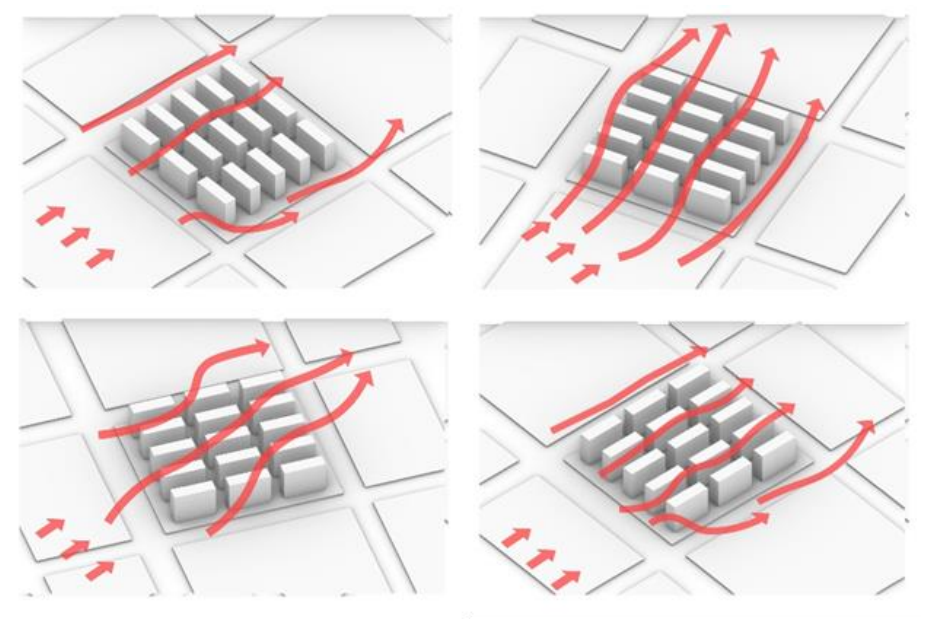

Figure 3. The flow of different wind directions under the spatial layout of the building. Source: Authors.

\subsubsection{Optimizing the vertical layout design of the building}

(1) Building height

From the perspective of the overall height of the building complex, the ventilation of the multi-story building layout is better than that of the mixed layout, and the worst is the wind environment of the highrise layout. Among the plots with the mixed layout of buildings, the plots with the mixed layout of multistory and small high-rise buildings have the best ventilation condition, followed by the mixed layout of multi-story, small high-rise, and high-rise buildings, and the worst ventilation condition is the mixed layout of multi-story buildings and high-rise buildings (Liu, 2011).

In general, the higher the building height, the higher the building's resistance to wind. The larger the area of the quiet wind area outside the rear building, the worse the ventilation; the lower the building height, 
the more the building's resistance to wind Low, the smaller the area of the quiet wind area of the rear building, the better the ventilation.

(2) The height arrangement form of the building

The different height arrangements between the buildings in the plot will have different effects on the wind environment. After the incoming wind passes through the front row of buildings on the windward side, its wind speed increases with the increase of the height of the building, and the area of the wind shadow area on the leeward side also increases (Liu, 2011). There are many kinds of height arrangements of the building group, which can be roughly divided into the same front and rear height type, front high back low type and front low back high type (here do not consider the height change of the east-west building group), various arrangements of different building heights Will lead to different wind environment conditions, as shown in Figure 4.
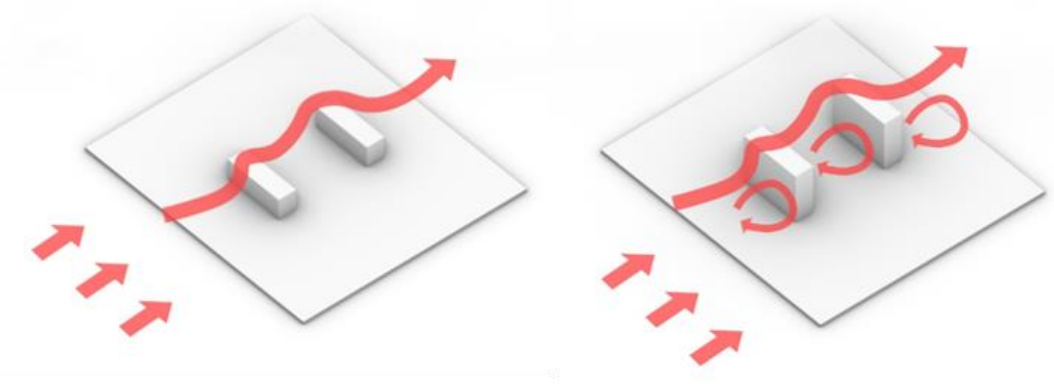

Figure 4. The diffusion method of airflow in the case of a multi-story building (left) and high-rise building (right) at the same front and rear heights. Source: Authors.

When the building complex has a multi-story layout, near the ground, when the incoming wind encounters the front row of buildings, the airflow will be blocked, resulting in a relatively small area of wind shadows on the back of the front row of buildings, and it also affects the back row of buildings. The influence of the wind environment is also minimal. There are many plots in the multi-story layout. When the distance between the front row of buildings and the rear row of buildings is longer than the length of the wind shadow area generated by the front row of buildings, the wind environment of the rear row of buildings is not affected; The distance between the rear buildings is shorter than the length of the wind shadow area generated by the front buildings, the wind speed on the outside of the windward side of the rear buildings is smaller, and the ventilation between the buildings is poor.

When the building group is high-rise and the front and rear heights of the buildings are the same, if the building spacing is not enough, the rear building will be in the wind shadow area of the front building, and the spatial wind environment will be the worst; if the building spacing is greater than that of the front building When the wind shadow area is large, the spatial wind environment of the rear buildings is less affected by the front buildings.

When a high-rise building is in the upwind direction of a multi-story building and the building group is a mixed multi-story and high-rise layout, the airflow passing through the front row of the high-rise building will increase the outdoor wind speed in front of the multi-story building during the sinking process. Mixing with the horizontal airflow on the windward side of a multi-story building will result in poor outdoor air circulation. If the length of the wind shadow area of a high-rise building is greater than the distance between a multi-story building and a high-rise building, it will have a great impact on the outdoor wind environment of the multi-story building, as shown in Figure 5.

When the building complex has a mixed layout of multi-story and high-rise buildings, and the front of the building is low and the rear is high, the front row of multi-story buildings has a small effect on the wind. If the distance between the front row of buildings and the rear row of buildings is greater than the length of the wind shadow area generated by the front row of buildings, the wind environment of the rear row

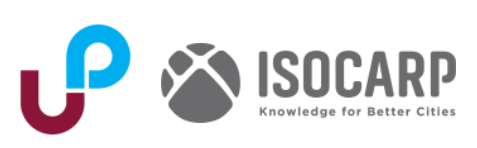


of buildings will basically not be affected. If the distance between the front row of buildings and the rear row of buildings is less than the length of the wind shadow area generated by the front row of buildings, the degree of influence of the outdoor wind environment of the rear row of buildings is related to the width and depth of the front row of buildings. The larger the width and depth of the building, the larger the wind shadow area (Liu, 2011).

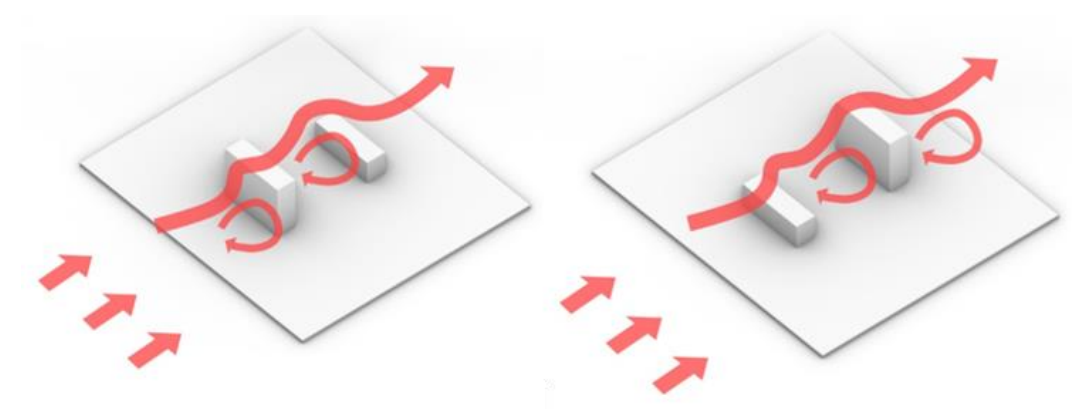

Figure 5. The diffusion method of airflow in different directions before and after multi-story and high-rise buildings. Source: Authors.

When designing the height of the buildings in the coastal area, to maximize the ocean airflow containing high concentrations of negative air ions to the inland, it is recommended that the height of the buildings in front of the windward be lower, and a small number of high-rise buildings are arranged in the inland areas far from the ocean. Buildings, the front-low-to-high building layout, and the spacing between the buildings are larger than the length of the wind shadow area of the front row of buildings, which will allow more ocean currents to enter the inland, thereby increasing the negative air ions in the plot (Chen, Zhang, 2010).

\subsubsection{Optimizing the distance to the ocean airflow}

Both the horizontal layout elements and the vertical layout elements of the building have a certain impact on the airflow. If the buildings in the offshore area block the airflow greatly, the air intake in the entire area will be greatly reduced. In this case, even if the area close to the ocean has a good spatial layout, it cannot change the air circulation in the area. Therefore, in the spatial layout, it is recommended that the space close to the ocean be laid out in a form with better ventilation (that is, combined with architectural layout elements, such as horizontal layout and vertical layout strategies). This will expand the initial air intake in areas far away from the ocean, increase the concentration of negative air ions in the area, and achieve the effect of improving regional air quality.

\section{References}

Chen, H. and Zhang, J.N. (2010) 'A Review of Researches on Influencing Factors of Air Anion Concentration Distribution', Ecological science, 29(02), p181-185.

Chu, J. (2010) 'Research on the Spatial Pattern of Urban Waterfront under the Health Orientation', Master Thesis, Harbin Institute of Technology, Harbin , Heilongjiang, China

Editor-in-Chief of Beijing Forestry College. (1999) Plant Physiology. Beijing: China Forestry Press.

Jia, P.S, Liu, S.P. and Wu, J.Y. (2020) 'Brief Introduction to the Research on the Correlation between Air Quality and the Morphological Characteristics of Residential Districts', Journal of Western Human Settlements and Environment, 35(06), p1-9.

Liu, J.P. (2011) Urban Environmental Physics. Beijing: China Construction Industry Press. 
Lin, J.M., Song, G.Q. and Zhao, L.X. (2006) Environment, health and negative oxygen ions. Beijing: Chemical Industry Press.

Li, J.G. and Lu, G.H. (2004) 'Research on the Construction of Urban Sports Life Circle', Sports research, 25(01), p5-6.

Meng, J.J., Zhang, Y. (2004) 'The Distribution Law of Air Anion Concentration on the Ground of Some Scenic Spots in Guangxi', Environmental Science Research, 17(03), p25-27.

Qian, F., Pan, K.X. and Xi, G.D. (2016) 'Preliminary Study on the Construction of Negative Ion Life Circle Facing Healthy City', Architecture and culture, (05), p192-193.

Wang, W. and Yu, Z. (2013) 'Research Progress of Air Anions in China's Urban Environment', Journal of Eco-Environment, 22(04), p705-711.

Wang, W., Yu, Z. and Zhen, F.Y. (2012) 'Distribution Characteristics of Air Anion Concentration in Different Environmental Locations in Summer and Its Relationship with Environmental Factors', Urban environment and urban ecology, 25(02), p38-40.

WHO Media centre. (2011) Air quality and health [online]. Available at: https://www.who.int/mediacentre/factsheets/fs313/zh/ (Accessed: 29 July 2021)

Yin, X. (2018) 'Research on City Height Control Strategy from the Perspective of Microclimate', Master Thesis, Dalian University of Technology, Dalian, Liaoning, China

Zhu, C.Y., Li, S.H. and Li, X.Y. (2012) 'The effect of canopy closure degree of urban green belt on air anion concentration and bacterial content', Chinese landscape architecture, 28(09), p72-77.

Zhang, J.C. (1994) 'The Formation of Negative Oxygen Ions in the Air and the Law of Its Concentration Decay', Journal of Textile Basic Science, 7(04), p306-318. 